\title{
Rosuvastatin cocrystals: an attempt to modulate physicochemical parameters
}

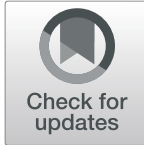

Venkata Deepthi Vemuri ${ }^{*}$ (D) and Srinivas Lankalapalli²

\begin{abstract}
Background: The meager physicochemical properties like low solubility and low dissolution rate of rosuvastatin calcium remain as an obstruction for formulation development. In the present work, we explore the evolution of rosuvastatin cocrystal, which may offer the synergetic physico-chemical properties of the drug. Cocrystal crafting depends on two possible intermolecular interactions; heteromeric and the homomeric selection of compounds with complementary functional groups are contemplated as a possible cause of supramolecular synthons in cocrystal formation. Specifically, cocrystals of rosuvastatin with L-asparagine and L-glutamine with molar ratio (1:1) were fabricated by using slow solvent evaporation and slow evaporation techniques. Novel cocrystals of rosuvastatin-asparagine (RSC-C) and rosuvastatin-glutamine (RSC-G) cocrystals obtained by slow solvent evaporation were utilized for preliminary investigation and further scale-up was done by using the solvent evaporation technique.

Results: The novel cocrystals showed a new characteristic of powder $X$-ray diffraction, thermograms of differential scanning calorimetry, ${ }^{1} \mathrm{H}$ liquid FT-NMR spectra, and scanning electron microscopy. These results signify the establishment of intermolecular interaction within the cocrystals. In both the novel cocrystals, rosuvastatin was determined to be engaged in the hydrogen bond interaction with the complementary functional groups of Lasparagine and L-glutamine. Compared with the pure rosuvastatin, RSC-C and RSC-G cocrystal showed 2.17-fold and 1.60-fold improved solubility respectively. The dissolution test showed that the RSC-C and RSC-G cocrystal exhibited 1.97-fold and 1.94-fold higher dissolution rate than the pure rosuvastatin in pH6.8 phosphate buffer respectively.

Conclusion: Modulation in the chemical environment, improvement in the solubility, and dissolution rate demonstrated the benefit of co-crystallization to improve the physicochemical properties of the drug.
\end{abstract}

Keywords: Rosuvastatin calcium, Cocrystals, L-asparagine, L-glutamine, Solvent evaporation

\section{Background}

Among all the physicochemical properties of the active moiety, dissolution and solubility are well deliberated to be the rate-determining step of poorly water-soluble drugs during oral absorption. Method selection for solubility and dissolution rate enhancement is crucial in attaining good oral absorption. Considering different methods, pharmaceutical cocrystals offers the most promising approach to improve physicochemical properties of the active moiety like solubility, melting point,

\footnotetext{
* Correspondence: deepthichowdary438@gmail.com

${ }^{1}$ Maharajah's College of Pharmacy, Phool Baugh, Vizianagaram 535002, India Full list of author information is available at the end of the article
}

dissolution, and stability $[1,2]$. Pharmaceutical cocrystals are multi-component complexes containing two or more molecules $[3,4]$. The main edge in cocrystal formation is the adjustment of physicochemical properties of drug molecules through alteration in its supramolecular structure, without a change in its covalent structure [5]. An important aspect in cocrystal crafting depends on two possible intermolecular interaction heteromeric and the homomeric selection of components with complementary functional groups that are contemplated as a possible cause of supramolecular synthons in cocrystal formation [6, 7]. The co-crystallization approach is a well productive choice that notably furnishes the active 
molecule with required physicochemical properties [8, 9] and also donates the right of intellectual property protection [10]. Furthermore, accompanying high capability without waste products and causing co-crystallization and an influential chunk of Green chemistry [11].

Preparation methods of cocrystals include precipitation, sublimation, solution crystallization, and solvent evaporation [12-14]. In the solvent-free technique, the intermittent phase is formed by co-grinding of the drug and co-former [15]. Along with the above approaches, various other techniques are also hired for the development of cocrystals such as super-critical fluids, sonic slurring, hot-melt extrusion, wet and dry compression, and ultrasound [16-18].

Rosuvastatin, (E,3R,5S)-7-(4-(4-fluorophenyl)-2-(methyl(methylsulfonyl)amino)-6-propan-2-ylpyrimidin-5-yl)-3,5-

dihydroxyhept-6-enoate has been utilized for the treatment of hyperlipoproteinemia, hypercholesterolemia, atherosclerosis, and avoidance of coronary heart diseases which belongs to BCS class-II drugs $[19,20]$. Rosuvastatin comes under the statin family, known as "super statin" [21, 22], which contains two oxydrilic group bound to asymmetric carbon atoms which are part of side-chain linked to a pyrimidine ring. The major trouble with this drug is low water solubility $(0.33 \mathrm{mg} / \mathrm{ml})$ which displays low solubility in gastrointestinal fluids [23]. Previously, various efforts to enhance the solubility of rosuvastatin such as polymerization [24], liquisolid technology [25], and nanoemulsifying delivery systems [26] were ample in the literature. Cocrystals of rosuvastatin were formulated by utilizing sorbitol [27] and vanillin [28] as co-formers. These cocrystal inventions provided the feasibility in utilizing the rosuvastatin cocrystals to modulate and design better product [29]. Ferrori et al. 2014 utilized three different rosuvastatin co-crystal such as Rosuvastatin 2-aminopyrimidine hemihydrate, Rosuvastatin pyrazine hydrate, and Rosuvastatin quinoxaline in the rosuvastatin synthesis process [30].

Amino acids are Zwitterionic compounds that exist under the GRAS group with low hazardous to the biological system in comparison with other co-formers [31, 32]. Moreover, their zwitterionic potentialities could form charge-assisted hydrogen bondings in cocrystal formation [33, 34]. Based on the synthon approach, cocrystal formation mainly depends upon the functional groups that are present in the drug molecule and the coformer [35, 36]. Carboxyl groups and amino groups present in amino acids act as good hydrogen bond acceptors and donors respectively, so these may be the satisfying co-former candidate for rosuvastatin cocrystal formulation.

In the present investigation, two amino acids containing the amide group, shown in Fig. 1, are preferred to form cocrystal with rosuvastatin. Specifically, cocrystals of RSC with L-asparagine and L-glutamine were

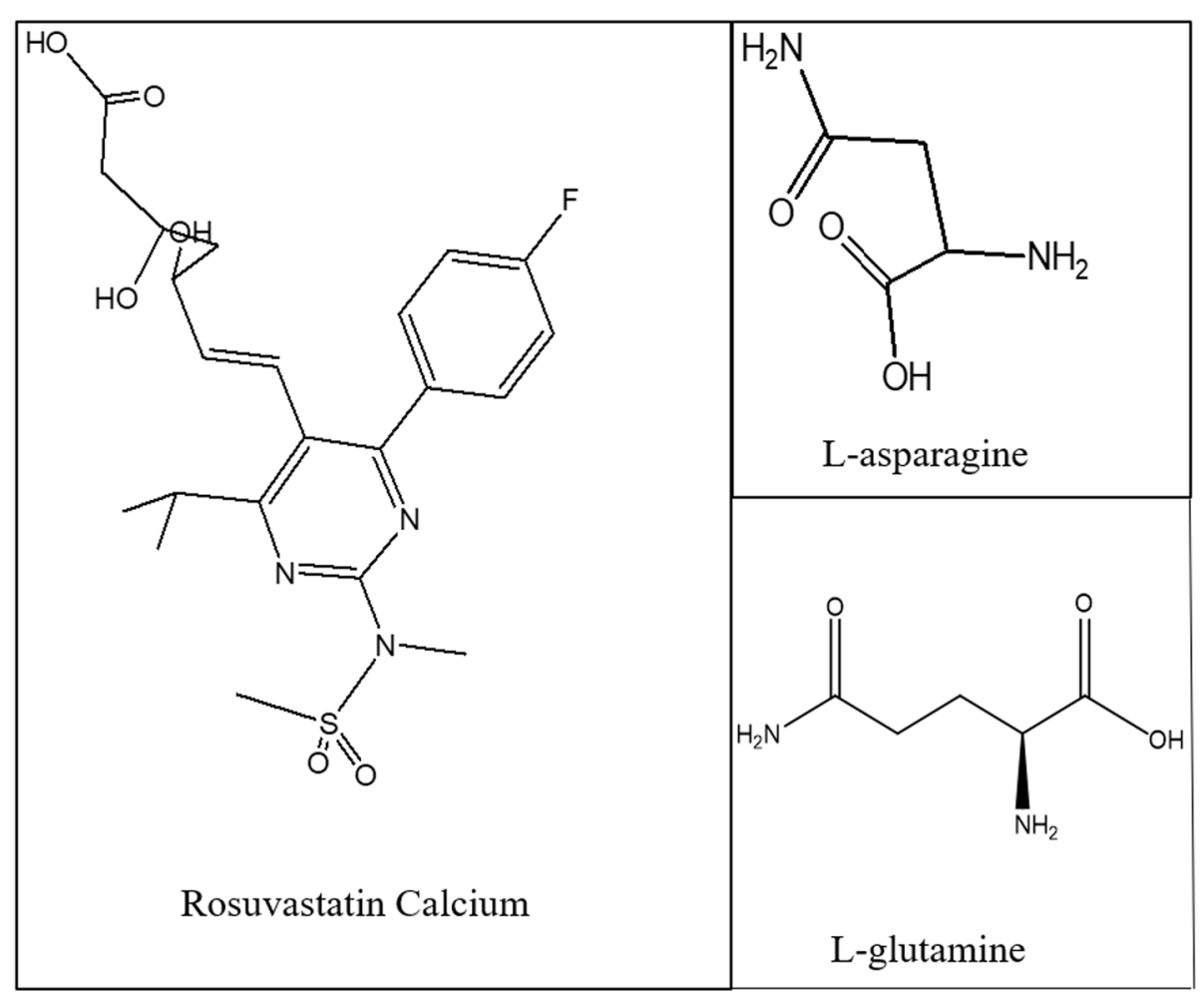

Fig. 1 Molecular structures of pure rosuvastatin calcium (RSC), L-asparagine (ASN), and L-glutamine (GLU) 
synthesized by the solvent evaporation technique. For the novel cocrystals, structural characterization was done by powder $\mathrm{x}$-ray diffraction (PXRD), infrared spectroscopy (FT-IR), differential scanning calorimetry (DSC), ${ }^{1} \mathrm{H}$ liquid FT-NMR, and scanning electron microscopy (SEM). In vitro evaluations were done by aqueous solubility and in vitro dissolution drug release and these were compared with the parent drug molecule.

\section{Methods}

\section{Materials}

Rosuvastatin calcium (RSC), L-asparagine, and L-glutamine were procured from Lotus chemicals (Andhra Pradesh, India). Methanol (chromatographic grade) was procured from Balda chemicals (Andhra Pradesh, India). All other chemicals utilized in the study were of commercial grade.

\section{Cocrystal designing}

Rosuvastatin is characterized by the presence of twooxydrilic groups bound to asymmetric carbon atoms which are part of a side chain linked to a pyrimidine ring, presented in Fig. 1. Considering rosuvastatin functional groups, co-former having an amide group may have the chance of forming hydrogen bonds. L-asparagine and L-glutamine are the amide-containing compounds and also Zwitterionic compounds were selected for the present study.

\section{Synthesis of novel cocrystals Slow solvent evaporation}

The cocrystals are produced by a molar ratio of $1: 1$ was as given: RSC (500 mg) with ASN (140 mg) and GLN (156 mg). The drug and the conformer were dissolved in a 3:1 ratio of ethanol and water in the test tube. The container was sealed with aluminium foil and 3 to 4 holes were stabbed on the foil for slow evaporation. The container was placed in the desiccator for 2 to 3 days and the cocrystals obtained were utilized for preliminary investigation.

\section{Solvent evaporation}

Two novel cocrystals of rosuvastatin calcium with L-asparagine and L-glutamine were prepared by the solvent evaporation technique. The cocrystals are produced by a molar ratio of $1: 1$ was as given: RSC (500 mg) with ASN (140 mg) and GLN (156 mg). The drug was dissolved in $15 \mathrm{ml}$ of methanol and the co-former in $5 \mathrm{ml}$ of distilled water. Both the solutions were stirred, and then ultrasonicated until the material was completely dissolved to form a clear solution. Methanol solution containing RSC was placed on the magnetic stirrer and the aqueous solution containing co-former was added dropwise. The stirring was continued until the solvent was completely evaporated. The product formed was placed in a desiccator overnight for complete drying of the cocrystals.

\section{Structural characterization \\ Fourier transform infrared spectroscopy analysis}

Fourier transform infrared spectroscopy spectra of RSC, ASN, GLU, RSC-C, and RSC-G is recorded using Azilent 360 series spectrometer containing DLATGS detector with $2 \mathrm{~cm}^{-1}$ spectral resolution. Then, 2-4 $\mathrm{mg}$ of each sample was placed on the sample cell and scanned through the wavelength of $4000-400 \mathrm{~cm}^{-1}$. The obtained data was analyzed using spectrum software.

\section{Differential scanning calorimetry analysis}

The thermal behavior of the samples was analyzed by utilizing differential scanning calorimetry. Each sample (4-5 mg) of pure drug, co-formers, and novel cocrystals were scanned by using Mettler Teledo DSC 8221e calorimetry. Each sample was sealed in aluminium sample pan, and an empty sealed aluminium pan was used as a reference. All the samples were scanned at a rate of 10 ${ }^{\circ} \mathrm{C} \min ^{-1}$ starting from 25 to $350{ }^{\circ} \mathrm{C}$ under nitrogen purge $(30 \mathrm{ml} / \mathrm{min})$.

\section{Powder X-ray diffraction analysis}

Crystalline structure alterations of RSC and novel cocrystals were analyzed by utilizing powder XRD diffractometer. Each sample of the pure drug, co-formers, and the cocrystals were placed in the sample holder then scanned by utilizing Philips Xpert MPD diffractometer with $\mathrm{Cu}$ target $\mathrm{X}$-ray tube source which was operated at $30 \mathrm{kV}$ and $15 \mathrm{~mA}$ for 2 scan axis. Maintaining a $5-65^{\circ}$ scan range with $0.02^{\circ}$ of step width and $10.00^{\circ} / \mathrm{min}$ scan speed.

\section{${ }^{1} \mathrm{H}$ liquid FT-NMR analysis}

${ }^{1} \mathrm{H}$ liquid FT-Nuclear Magnetic resonance spectra for RSC, ASN, GLU, RSC-C, and RSC-G is recorded by utilizing $400 \mathrm{MHz}$ FT-NMR spectrometer (JNM-ECZ 400S) USINF TMS as an internal standard. Each sample was dissolved in deuterated dimethyl sulfoxide (DMSO- $\mathrm{d}_{6}$ ) for analysis and operated at $400 \mathrm{MHz}$ frequency. The obtained spectra were integrated using JEOL Delta software.

\section{Scanning electron microscopy analysis}

Morphological changes between the pure drug RSC and the novel cocrystals were analyzed with scanning electron microscopy. Scanning electron microscopy (SEM) analysis was done by using XL30ESEM with EDAX containing backscattered and secondary electron detectors. The particles were prepared for the analysis by placing on aluminium stabs and coated with gold under vacuum. 
The samples were photomicrographed at different magnifications.

\section{In vitro evaluation \\ Apparent solubility}

The equilibrium method [37] was utilized for determining the apparent solubility of pure drug RSC, cocrystals RSC-C, and RSC-G. An excess amount of each sample was placed in vials with caps containing $10 \mathrm{ml}$ of $\mathrm{pH} 6.8$ phosphate buffer. These solutions were sonicated for $15 \mathrm{~min}$ and were placed in a water bath shaker for $48 \mathrm{~h}$ with a rotating speed of 200 $\mathrm{rpm}$ at $30 \pm 1{ }^{\circ} \mathrm{C}$. The supernatant solution was filtered through $0.45 \mu \mathrm{m}$ filters. This filtrate was diluted with methanol and the drug concentration was determined by utilizing UV-Spectrophotometer (V-630, Jasco. Japan) at $244 \mathrm{~nm}$. Apparent solubility analysis was conducted in triplicate and the residual solids were qualitatively analyzed by Fourier transform infrared spectroscopy (FT-IR).

\section{In vitro dissolution study}

The in vitro drug release studies were carried out triplicate in Erweka DT600 USP apparatus-II (paddle method). The highest dose of RSC (40 mg), RSC-C, and RSC-G (Equivalent to $40 \mathrm{mg}$ of pure drug) was put into $900 \mathrm{ml}$ of dissolution media. The paddles were rotated at $100 \mathrm{rpm}$ by maintaining $37 \pm 0.5{ }^{\circ} \mathrm{C}$ throughout the study. Two milliliters of aliquot was collected at 10, 20, 40,80 , and 120 by replacing with fresh dissolution medium. Collected aliquots were filtered through a 0.45 $\mu$ filter; the filtrate was diluted and estimated for drug concentration using UV-Spectrophotometer (V-630, Jasco. Japan) at $244 \mathrm{~nm}$.

\section{Results}

\section{Structural characterization}

\section{Fourier transform infrared spectroscopy}

Alterations of intermolecular interactions in comparison to parent molecules can be studied using FT-IR [38]. Figure 2 shows the FT-IR spectra of starting materials and novel cocrystals. For RSC, the characteristic peaks corresponding to carboxylic $\mathrm{O}-\mathrm{H}$ stretch and $\mathrm{N}-\mathrm{H}$ stretch are assigned at $3382,2968 \mathrm{~cm}^{-1}$ respectively [39]. For ASN, a sharp peak appeared at $3378 \mathrm{~cm}^{-1}$ was assigned to $\mathrm{O}-\mathrm{H}$ stretch, and a broad multiple peaks at 3099 was ascribed as N-H stretch [40]. For GLN, a characteristic $\mathrm{O}-\mathrm{H}$ peak was assigned at 2401, and a broad multiple peaks corresponding to $\mathrm{N}-\mathrm{H}$ stretch was ascribed at $3168 \mathrm{~cm}^{-1}[41,42]$. These are the main functional groups that are responsible for hydrogen bond formation.

The FTIR analysis RSC-C cocrystals showed the shift in the $\mathrm{O}-\mathrm{H}$ peak position of parent molecule from 3382

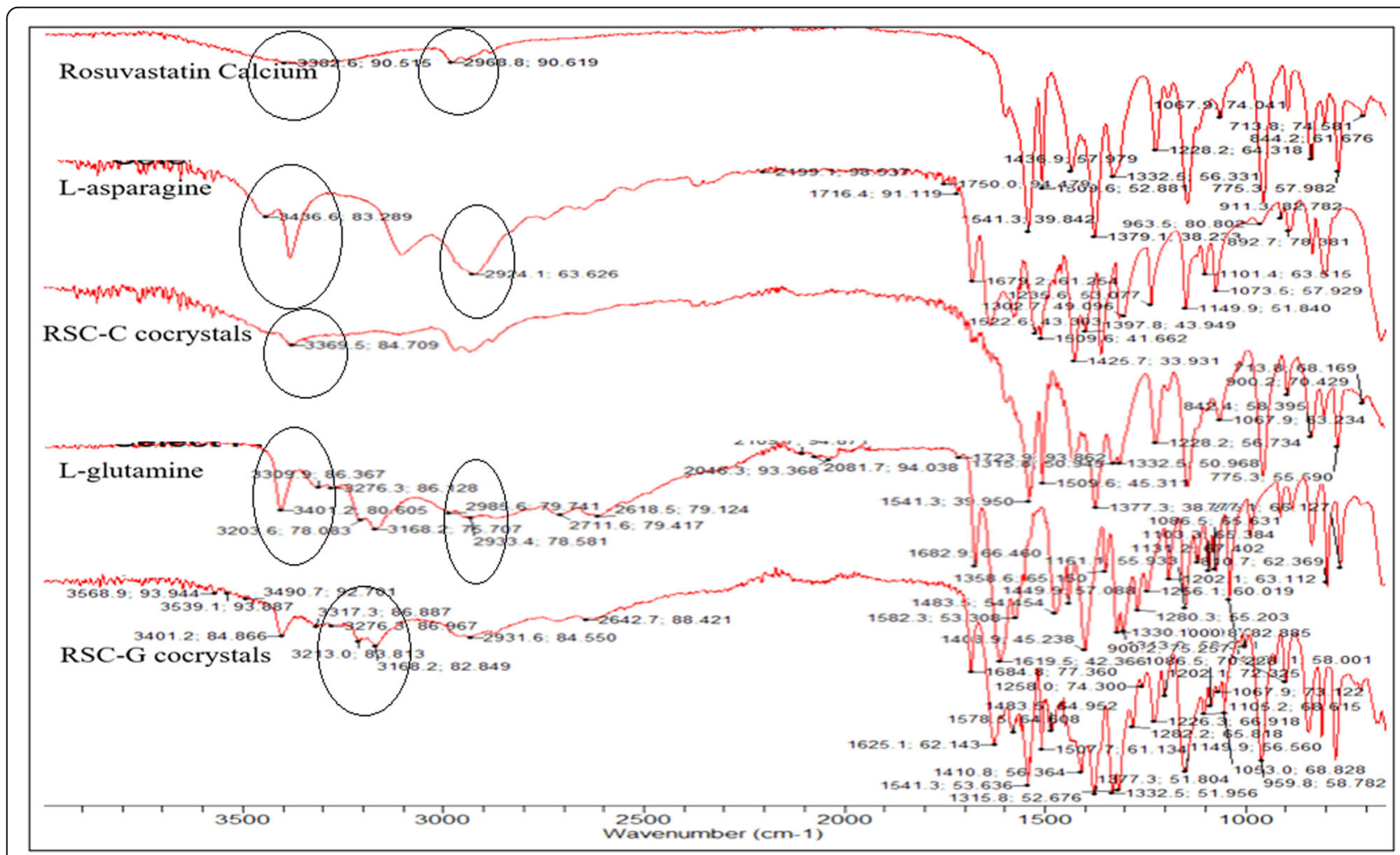

Fig. 2 FTIR spectra of RSC, ASN, GLN, RSC-C, and RSC-G 
$\mathrm{cm}^{-1}$ to and nitrogen peak of ASN from 3099 to 3369 $\mathrm{cm}^{-1}$; meanwhile, the carbonyl group shift from 1654 to 1686. RSC-G cocrystals exhibited a shift in the O-H peak of RSC from 3382 and nitrogen peak of GLN from 3213 to 3401; meanwhile, the carbonyl group shifted from 1654 to 1684 . A similar type of shift can be noticed in both the novel cocrystals indicating a similar type of interaction.

\section{Differential scanning calorimetry}

The cocrystals of RSC-C and RSC-G formulated by the solvent evaporation technique were analyzed for their thermal behavior concerning the starting compounds. Differential scanning calorimetry (DSC) was widely used in investigating the compatibility status and structural alterations in the molecule [43]. DSC thermograms of the starting material and novel cocrystals were shown in Fig. 3. RSC presented an endothermic peak between 65 and $80{ }^{\circ} \mathrm{C}$, which is considered as a water loss peak, to the next RSC showed multiple endothermic melting peaks between 230 and $300{ }^{\circ} \mathrm{C}$ displaying the semicrystalline structure of the molecule [44]. Co-formers ASN and GLN showed a sharp endothermic melting peak at $241{ }^{\circ} \mathrm{C}$ and $193{ }^{\circ} \mathrm{C}$ respectively.

In RSC-C novel cocrystal broad endothermic between 60 and $80{ }^{\circ} \mathrm{C}$ indicates the lattice water existence and a sharp endothermic event at $129{ }^{\circ} \mathrm{C}$ ascribed as the melting point which is a major alteration when compared to the parent molecule. RSC-G cocrystal presented a sharp endothermic peak at $198{ }^{\circ} \mathrm{C}$ indicating the melting point deviation with the parent molecule. In both the cocrystal, the multiple endothermic melting peaks of the parent molecule have vanished, confirming the loss of semi-crystalline nature of the compound.

\section{Powder X-ray diffraction}

Most of the drugs exhibit various forms in their solidstate like crystalline, amorphous polymorphs, and solvates. In turn, these forms can vary extensively in their physicochemical properties [45]. Along with the DSC, powder X-ray diffraction (PXRD) analysis has the ability to determine the crystallinity of the drug molecule. PXRD spectra of the starting materials and the novel cocrystals were shown in Fig. 4. PXRD pattern of RSC indicated the semi-crystalline nature of the molecule, which is in agreement with the previous literature [44].

RSC-C cocrystal showed the major characteristic peaks of $2 \Theta$ scattering angles at $9.14^{\circ}, 10.28^{\circ}, 20.85^{\circ}, 24.61^{\circ}$, $32.93^{\circ}$, and $40.06^{\circ}$ which were absent in the drug molecule and differ from co-former. RSC-G showed the major characteristics peaks of $2 \Theta$ scattering angles at $20.61^{\circ}, 23.58^{\circ}, 24.20^{\circ}, 26.74^{\circ}, 29.41^{\circ}, 3.58^{\circ}, 34.98^{\circ}, 39.39^{\circ}$, and $40.95^{\circ}$ which are new for RSC and differ from GLN.

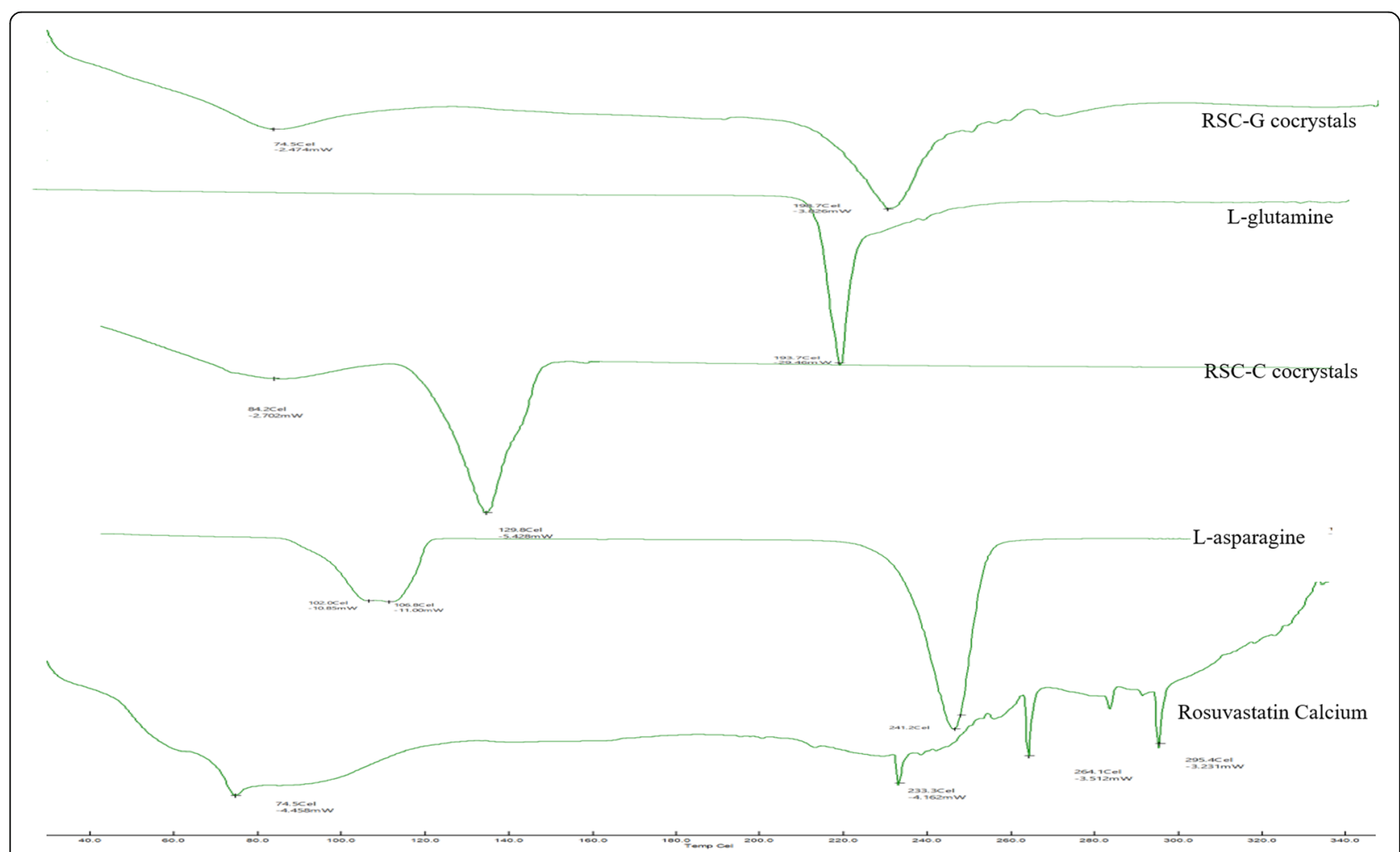

Fig. 3 DSC thermogram of RSC, ASN, GLN, RSC-C, and RSC-G 


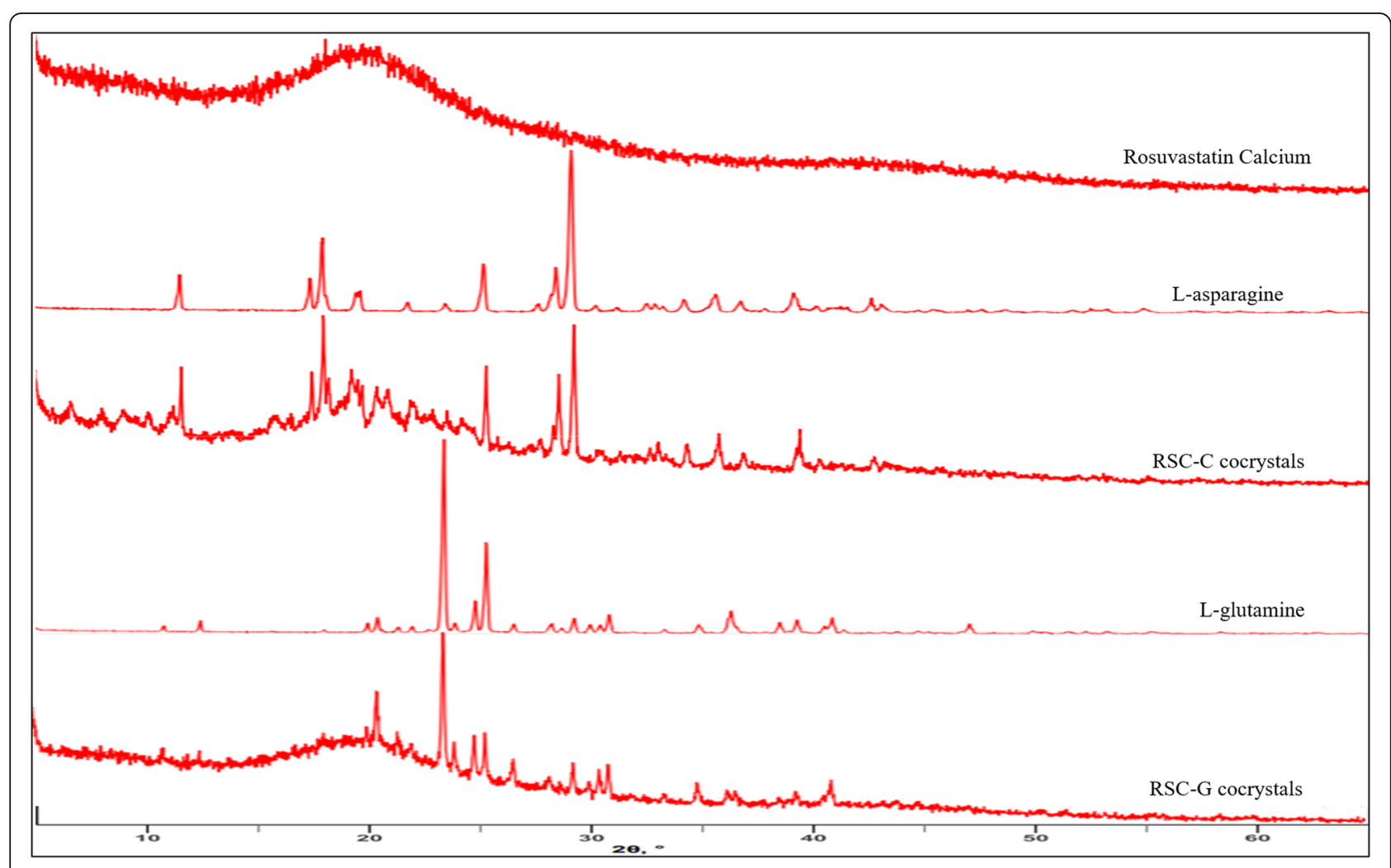

Fig. 4 Powder X-ray diffraction pattern of RSC, ASN, GLN, RSC-C, and RSC-G

\section{${ }^{1} H$ liquid FT-NMR}

Alteration in the chemical environment can be readily detected through the chemical shift in NMR spectrum. Both the Novel cocrystals, RSC-C and RSC-G, showed the co-existence of resonance peaks from the starting materials with significant chemical shift indicating the new phase generation [46]. A solvent peak (DMSO- $\mathrm{d}_{6}$ ) at $\delta=2.6$ can be detected in both the starting materials and the co-crystals. Plain RSC showed the proton peaks at $\delta=1.16,2.10,3.50,3.35,3.71,6.45,7.22$, and 7.65 , in which 3.35 and 3.71 are assigned to protons of two oxydrilic groups which are considered to be the main characteristic groups that involved in hydrogen bond interaction. All the proton peaks assigned were clearly indicated in Fig. 5. RSC-C cocrystals showed a major peak shift in these oxydrilic groups to 2.65 and 3.68 respectively. Whereas, RSC-G cocrystal exhibited the chemical shift in oxydrilic groups to 3.32 and 3.69 respectively.

\section{Scanning electron microscopy}

The habit of the starting materials and the novel cocrystals prepared from solvent evaporation as shown in Fig. 6. Plain RSC presented as plate-shaped particles with irregular surface morphology and random sizes. Co-formers ASN and GLN appeared as stick-shaped crystalline and with smooth surface morphology. In RSC-C cocrystals, the plate-shaped particles were converted to an irregular closely fitted crystalline structure. Whereas the RSC-G exhibited as stick-shaped closely fitted clusters. This alteration within the habit of the cocrystals might be due to the intermolecular hydrogen bonds between the drug and the co-former.

\section{In vitro evaluation \\ Apparent solubility}

The apparent solubility analysis was assessed for pure drug and the novel cocrystals in $\mathrm{pH} 6.8$ phosphate buffer. Quantification of RSC was assessed by UV spectrophotometer, and the outcomes are presented in the Table 1 . The samples were assessed after $4 \mathrm{~h}, 24 \mathrm{~h}$, and $48 \mathrm{~h}$ as described in the "Methods" section. The solubility enhancement of the cocrystals was reduced after $4 \mathrm{~h}$. The solubility of RSC cocrystals was presented to be very less after $24 \mathrm{~h}$, and no solubility enhancement was seen after $48 \mathrm{~h}$. This reduction may be due to the breakdown of cocrystals into its parent molecules.

Apparent solubility of novel cocrystals was compared with that of pure drug RSC. Plain RSC showed the solubility of $1.427 \mathrm{mg} / \mathrm{ml}$, whereas RSC-C cocrystals exhibited $3.466 \mathrm{mg} / \mathrm{ml}$ which is almost 2.17 -fold higher to that of the parent molecule, followed by RSC-G 

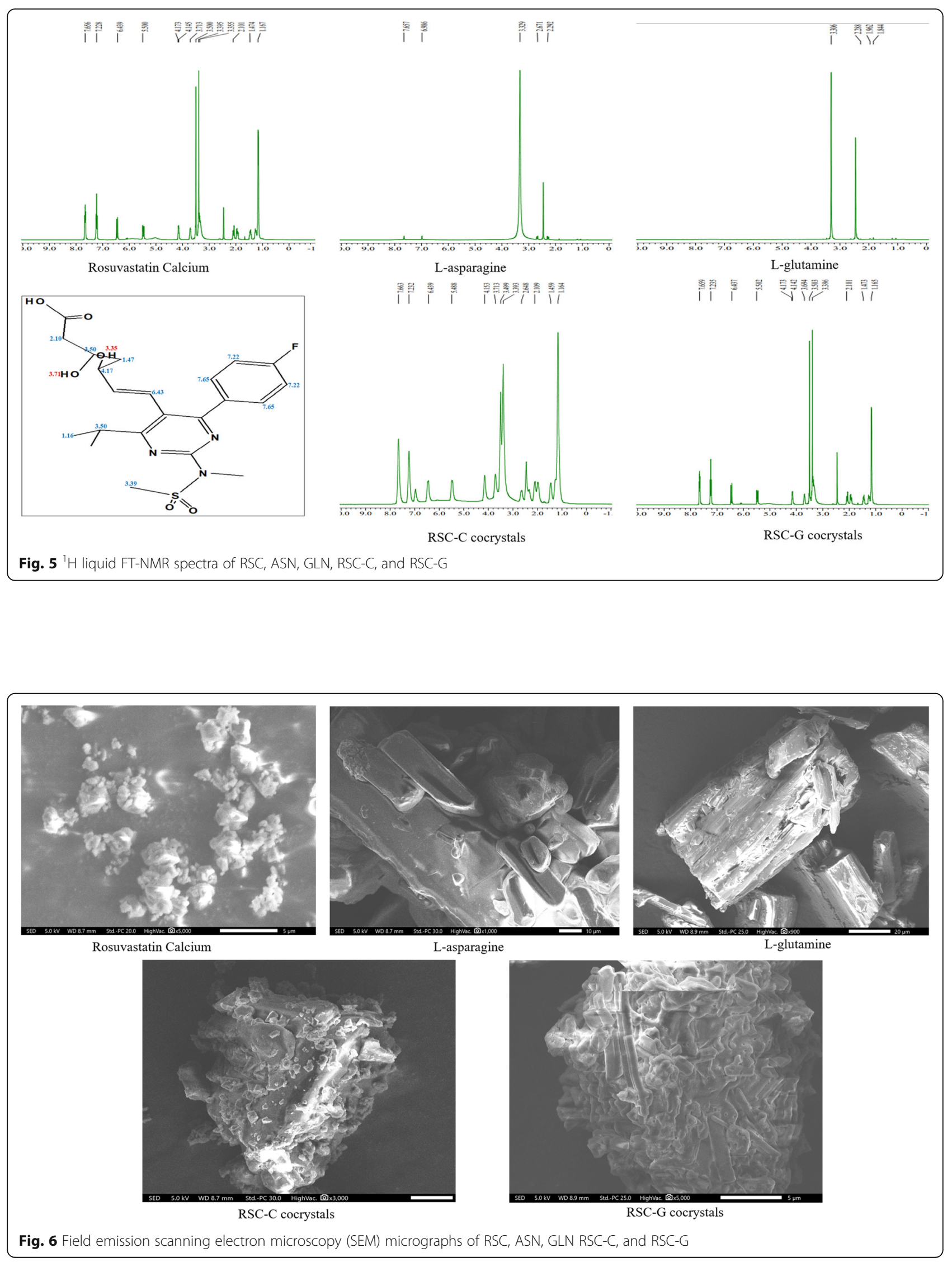
Table 1 Apparent solubility of rosuvastatin calcium

\begin{tabular}{lll}
\hline $\begin{array}{l}\text { Chemical } \\
\text { moiety }\end{array}$ & $\begin{array}{l}\text { Solubility in } \mathbf{~ p H} \text { 6.8 phosphate } \\
\text { buffer }(\mathbf{m g} / \mathbf{m l})\end{array}$ & Enhancement \\
\hline RSC & $1.427 \pm 0.034$ & - \\
RSC-C & $3.466 \pm 0.057$ & 2.17 -fold \\
RSC-G & $2.283 \pm 0.066$ & 1.64 -fold \\
\hline
\end{tabular}

Mean \pm SD, $n=3$

cocrystals that showed $2.283 \mathrm{mg} / \mathrm{ml}$ which is 1.64 -fold higher to parent molecule within $4 \mathrm{~h}$ (Smax) of the solubility analysis. The residual material was analyzed by FTIR after $4 \mathrm{~h}, 24 \mathrm{~h}$, and $48 \mathrm{~h}$ to find any alterations in cocrystals. These results showed that there is no change in cocrystals up to $4 \mathrm{~h}$; after $24 \mathrm{~h}$, the FT-IR spectra of the residual materials find similar to that of the pure drug [47] which was shown in Fig. 7.

\section{In vitro dissolution studies}

The in vitro drug release profiles of RSC, RSC-ASN physical mixture, RSC-GLN physical mixture, RSC-C, and RSC-G were analyzed in $\mathrm{pH} 6.8$ phosphate buffer and were shown in Fig. 8. In the media, the plain RSC, RSC-ASN physical mixture, and RSC-GLN physical mixture exhibited almost similar dissolution with $23.4 \%$ to $49.5 \%, 25.6 \%$ to $52.4 \%$, and $24.9 \%$ to $50.8 \%$ drug release in $120 \mathrm{~min}$ respectively, which is in agreement with the previous literature [44]. Whereas RSC-C and RSC-G cocrystals showed fastened and almost maximum drug release (>80\%) within $120 \mathrm{~min}$ of the study. RSC-C cocrystals exhibited $91.4 \%$ to
$97.7 \%$ of the drug release within $120 \mathrm{~min}$, which is almost 1.97-fold higher than that of pure drug. RSC-G cocrystals showed $84.6 \%$ to $86.3 \%$ of the drug release which is of 1.74-fold enhancement of drug release in comparison to pure drug. The studies pointed out the increased dissolution performance of RSC upon cocrystallization with L-asparagine and L-glutamine, which is in good agreement with apparent solubility studies.

\section{Discussion}

Cocrystals are cognominated among the products that are obtained from the crystal engineering approach with ionic/non-covalent intermolecular interaction between two or more disparate molecules with a certain stoichiometric ratio in a crystal lattice $[48,49]$ at most one molecule should be active moiety. Generally, the choice of co-former should be done from GRAS (Generally recognized as safe) or EAFUS (Everything added to food in united states) list, which is frequently without pharmacological efficacy $[50,51]$. Therefore, it contributes a practical approach in organizing the physicochemical properties with no medical efficacy diversity $[52,53]$. The co-former selected should essentially carry a group able to develop molecular synthons with the active moiety. In this framework, amino acids appear to be a beneficial option as an applicable companion. Amino acids exist under the GRAS group, besides pretty low toxicity and inexpensive. Many of the salt, that is formed from

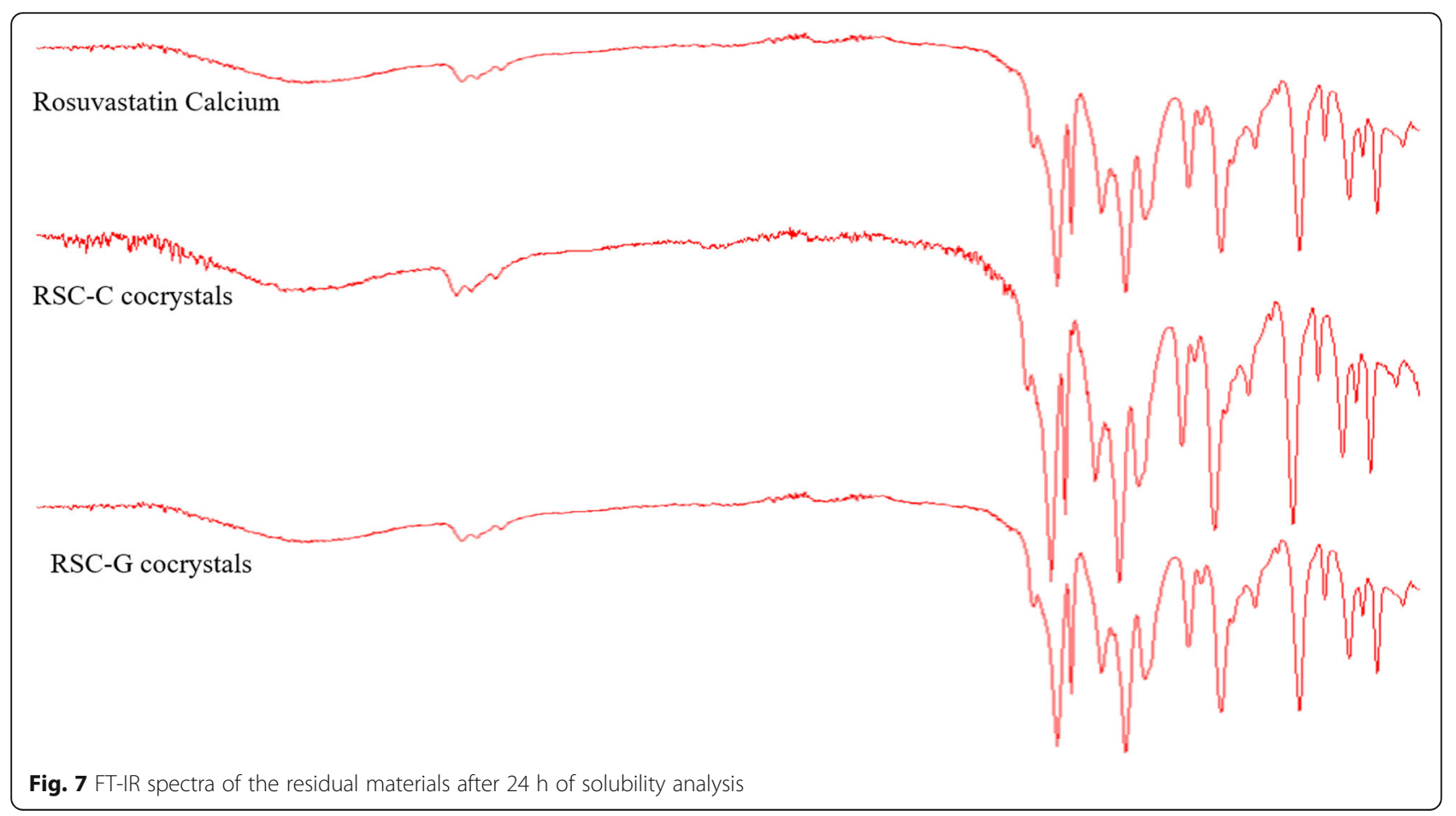




\section{In vitro dissolution studies}

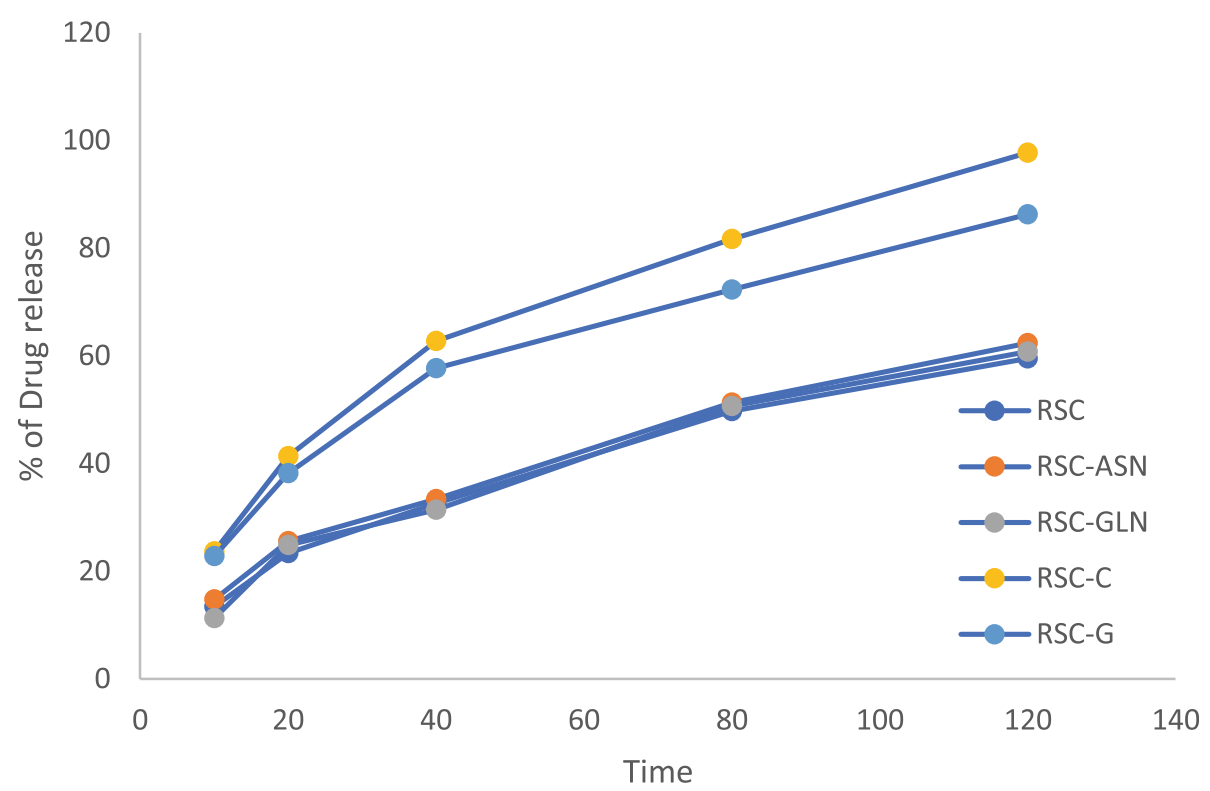

Fig. 8 In vitro dissolution studies of RSC, RSC-ASN physical mixture, RSC-GLN physical mixture, RSC-C, and RSC-G

the amino acids with various classes of therapeutic agents, were reported in previous literatures $[54,55]$.

The shift in the wavenumber points out the new chemical interactions of the drug and the co-former that impact the functional group position. Both the novel cocrystals show the $\mathrm{O}-\mathrm{H}$, which was converted to a broad single peak, and the nitrogen peak of the co-former was comparatively suppressed. This outcome confirms the merging of two functional groups through hydrogen bond formation between the hydroxyl group of the drug and nitrogen group of the co-former.

Based on the study of Perlovich [56], 55.3\% of cocrystals exhibit the melting point in the middle, $28.9 \%$ of cocrystals with low, and $14.5 \%$ exhibit higher endotherm than the starting materials. RSC-C presented a melting point at $129{ }^{\circ} \mathrm{C}$, which melts at the temperature below the melting point of the drug and the co-former. This decrease in enthalpy and melting point in comparison to pure compounds indicates the formation of the weak crystalline structure $[57,58]$. RSC-G presented a melting point at $198{ }^{\circ} \mathrm{C}$ indicating the melting point of cocrystal is in the middle of the drug and the co-former, which is a very common phenomenon in 1:1 stoichiometric cocrystals [56].

In DSC study, the nature of the novel cocrystal is represented by the position and shape of endothermic melting peaks. The sharp and narrow endothermic peaks presented by novel cocrystal indicate a high degree of purity and crystallinity of formed non-covalent derivatives [59]. The shift in the endothermic event within the formulation due to the alteration in the crystalline geometry of RSC induced by the interaction of free hydroxyl groups of RSC with the amine group of co-former. However, the presentation of peak distinctly indicates the absence of ionic interaction between the drug and the performer.

In PXRD analysis, the appearance of the new characteristic peaks in the novel cocrystals indicates the new phase generation. PXRD results confirm the conversion of the semi-crystalline nature of the drug into a new weak crystalline form in both the cocrystals. This new phase may be the cocrystal formation due to the hydrogen bond interaction between the drug and the coformer which was found to be in accordance with DSC and FT-IR studies.

Apparent solubility of novel cocrystals was compared with that of pure drug RSC. Plain RSC showed the solubility of $1.427 \mathrm{mg} / \mathrm{ml}$, whereas RSC-C cocrystals exhibited $3.466 \mathrm{mg} / \mathrm{ml}$ which is almost 2.17 -fold high to that of the parent molecule, followed by RSC-G cocrystals showed $2.283 \mathrm{mg} / \mathrm{ml}$ which is 1.64 -fold high to parent molecule with within $4 \mathrm{~h}(\mathrm{Smax})$ of the solubility analysis.

In FT-NMR analysis, both the cocrystal showed the downfield nature of chemical shift values indicating the involvement of both oxydrilic groups in bond formation. This chemical shift value alteration confirmed the cocrystal formation between drug and the co-former probably due to the interaction of free hydroxyl groups of drug with co-formers. Furthermore, the outcomes 
attended by the FT-NMR analysis were in good agreement with DSC, PXRD, and FT-IR analysis.

This enhancement of solubility until $4 \mathrm{~h}$ is sufficient to increase the absorption of the drug. This mechanism is known as the spring and parachute effect showed by many of the cocrystals $[60,61]$. According to this mechanism, after breaking down of cocrystals (after $4 \mathrm{~h}$ ), the more soluble part (co-former) of cocrystal is drained out of the crystal lattice into the solution. While the drug component evolves supersaturated in biological medium. This increase in energy of the drug molecule is known as spring energy. This condition lasts for sufficient time (parachute effect) to facilitate proper absorption.

In in-vitro dissolution analysis, RSC-C and RSC-G cocrystals showed fastened and almost maximum drug release $(>80 \%$ ) within $120 \mathrm{~min}$ of the study. RSC-C cocrystals exhibited $91.4 \%$ to $97.7 \%$ of the drug release within $120 \mathrm{~min}$, which is almost 1.97-fold higher than that of pure drug. RSC-G cocrystals showed $84.6 \%$ to $86.3 \%$ of the drug release which is of 1.74 -fold enhancement of drug release in comparison to pure drug. The studies pointed out the increased dissolution performance of RSC upon co-crystallization with L-asparagine and L-glutamine, which is in good agreement with apparent solubility studies. The strategy of converting the idea of cocrystals into the application was well explained in the previous literatures, which may be helpful for process development in the future investigation [62-64].

\section{Conclusion}

Although extensive research was dedicated to cocrystals, most of the literature reported novel cocrystal production and its modification in physicochemical properties; there is a lack of information regarding the selection of co-former and their effect on the biological system. Hence, the present study showed amino acids that exist under the GRAS group with low hazardous to the biological system as a suitable choice as co-former in cocrystal preparation. These amino acids, due to their zwitterionic potentialities, act as good hydrogen bond acceptors and donors. This study has demonstrated the formulation of rosuvastatin cocrystals through both L-asparagine and L-glutamine as the co-formers. The cocrystal structure alteration is compared with the spectral data of the parent molecule, implying the intermolecular interaction established within the cocrystals. In both cases, RSC was determined to be engaged in the hydrogen bond interaction with the complementary functional groups of ASN and GLN. Improvement in the solubility studies demonstrated the benefit of co-crystallization to improve the physicochemical properties. Furthermore, the outcomes of solubility analysis are in concurrence with the in vitro dissolution study. However, in the present investigation, the in vivo performance of the cocrystals like $C_{\max }$ and AUC have not been covered and will be taken up in our next publication. The outcomes clearly indicate the modulation in the chemical structure, solubility enhancement, and dissolution enhancement of RSC through co-crystallization.

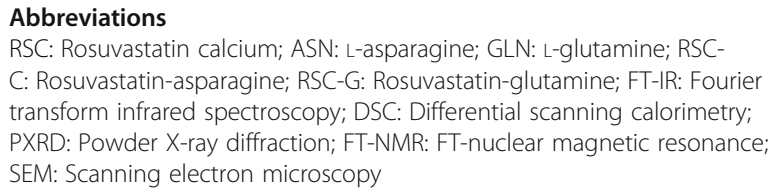

\section{Acknowledgements}

The authors are thankful to Maharajah's college of pharmacy, Vizianagaram, Andhra Pradesh and Gitam deemed to be university for providing the facilities to carry the research work.

\section{Authors' contributions}

VDV: synthesis, formulation development, plan of investigation, and literature review. SL: plan of investigation, supervision, and corrections. All the authors have read and approved the manuscript.

\section{Funding}

This research received no specific grant from any funding agency in public, commercial, or not-for-profit sector.

\section{Availability of data and materials}

The data generated or analyzed during this study are included in this article, if any excess data required will be available from the corresponding author on reasonable request.

\section{Declarations}

Ethics approval and consent to participate Not applicable.

\section{Consent for publication}

Not applicable.

\section{Competing interests}

The authors declare that they have no competing interest.

\section{Author details}

${ }^{1}$ Maharajah's College of Pharmacy, Phool Baugh, Vizianagaram 535002, India. ${ }^{2}$ GITAM Institute of Pharmacy, GITAM (Deemed to be University), Visakhapatnam, Andhra Pradesh 530045, India.

Received: 5 December 2020 Accepted: 21 February 2021

Published online: 12 March 2021

\section{References}

1. Maestrelli F, Cirri M, Mennini N, Zerrouk N, Mura P (2011) Improvement of oxaprozin solubility and permeability by the combined use of cyclodextrin, chitosan, and bile components. Eur J Pharm Biopharm 78:385-393

2. Almansa C, Mercè R, Tesson N, Farran J, Tomàs J, Plata-Salamán CR (2017) Cocrystal of tramadol hydrochloride-celecoxib (ctc): a novel API-API Cocrystal for the treatment of pain. Cryst Growth Des 17:1884-1892

3. Braga D, Grepioni F (2000) Intermolecular interactions in nonorganic crystal engineering. Acc Chem Res 33:601-608

4. Etter MC (1991) Hydrogen bonds as design elements in organic chemistry. J Phys Chem 95:4601-4610

5. Dai XL, Chen JM, Lu TB (2018) Pharmaceutical cocrystallization: an effective approach to modulate the physicochemical properties of solid-state drugs. Cryst Eng Comm 20:e5292-e5316

6. Oaki Y (2017) Morphology design of crystalline and polymer materials from nanoscopic to macroscopic scales. Bull Chem Soc Jpn 90:776-788

7. Lusi M (2018) Engineering crystal properties through solid solutions. Cryst Growth Des 18:3704-3712 
8. Thakuria R, Sarma B (2018) Drug-drug and drug-nutraceutical cocrystal/salt as alternative medicine for combination therapy: a crystal engineering approach. Crystals 8:101

9. Ueda A (2017) Development of novel functional organic crystals by utilizing proton- and $\pi$-electron-donating/accepting abilities. Bull Chem Soc Jpn 90: 1181-1188

10. Aakery CB, Salmon DJ (2005) Building co-crystals with molecular sense and supramolecular sensibility. Cryst Eng Comm 72:439-448

11. Cannon AS, Warner JC (2002) Noncovalent derivatization: green chemistry applications of crystal engineering. Cryst Growth Des 2:255-257

12. Aher S, Dhumal R, Mahadik K, Paradkar A, York P (2010) Ultrasound assisted cocrystallization from solution (USSC) containing a non-congruently soluble cocrystal component pair: Caffeine/maleic acid. Eur J Pharm Sci: Official J Eur Federation Pharm Sci 41:597-602

13. Hickey MB, Peterson ML, Scoppettuolo LA, Morrisette SL, Vetter A, Guzmán H, Remenar JF, Zhang Z, Tawa MD, Haley S, Zaworotko MJ, Almarsson Ö (2007) Performance comparison of a co-crystal of carbamazepine with marketed product. Eur J Pharm Biopharm 67:112-119

14. Weyna DR, Shattock T, Vishweshwar P, Zaworotko MJ (2009) Synthesis and structural characterization of cocrystals and pharmaceutical cocrystals: mechanochemistry vs slow evaporation from solution. Cryst Growth Des 9 : 1106-1123

15. Moradiya H, Islam MT, Woollam GR, Slipper IJ, Halsey S, Snowden MJ, Douroumis D (2014) Continuous cocrystallization for dissolution rate optimization of a poorly water-soluble drug. Cryst Growth Des 14:189-198

16. Berry DJ, Seaton CC, Clegg W, Harrington RW, Coles SJ, Horton PN, Hursthouse MB, Storey R, Jones W, Friscic T, Blagden N (2008) Applying hotstage microscopy to co-crystal screening: a study of nicotinamide with seven active pharmaceutical ingredients. Cryst Growth Des 8:1697-1712

17. James SL, Adams CJ, Bolm C, Braga D, Collier P, Friščić T, Grepioni F, Harris HKDM, Jones W, Krebs A, Mack J, Maini L, Orpen AG, Parkin IP, Shearouse WC, Steed JW, Waddell DC (2012) Mechanochemistry: opportunities for new and cleaner synthesis. Chem Soc Rev 41:413-447

18. Childs SL, Rodríguez-Hornedo N, Reddy LS, Jayasankar A, Maheshwari C, McCausland L, Shipplett R, Stahly BC (2008) Screening strategies based on solubility and solution composition generate pharmaceutically acceptable cocrystals of carbamazepine. Cryst Eng Comm 10:856-864

19. Potur RG, Moisuc LS, Saraficeanu N, Boita T, Macovei L, Telisça AD (2014) Stable pharmaceutical composition comprising amorphous rosuvastatin calcium. EP 2805714 A1.

20. Lusis AJ (2000) Atherosclerosis. Nature 407:233-241

21. Ballantyne MC, Miller E, Chitra R (2004) Efficacy and safety of rosuvastatin alone and in combination with cholestyramine in patients with severe hypercholesterolemia: A randomized, open-label, plulticenter trial. Clin Therapeut 26:1855-1864

22. Kostapanos SM, Derdemezis SC, Filippatos DT, Milionis JH, Kiortsis ND, Tselepis DA (2008) Effect of rosuvastatintreatment onplasma visfatinlevels in patients with primary hyperlipidemia. Eur J Pharmacol 578:249-252

23. Alshora DH, Haq N, Alanazi FK, Ibrahim MA, Shakeel F (2016) Solubility of rosuvastatin calcium in different neat solvents at different temperatures. J Chem Thermodyn 94:230-233

24. Sarfraz RM, Ahmad M, Mahmood A, Akram MR, Abrar A (2017) Development of b- cyclodextrin-based hydrogel microparticles for solubility enhancement of rosuvastatin: An in vitro and in vivo evaluation. Drug Des Dev Ther 11: 3083-3096

25. Lennernas H, Fager G (1997) Pharmacodynamics and pharmacokinetics of the HMG-21 CoA reductase inhibitors, similarities and differences. Clin Pharmacokinet 32:403-425

26. Balakumar K, Raghavan CV, Selvan NT, Prasad RH, Abdu S (2013) Self nanoemulsifying drug delivery system (SNEDDS) of rosuvastatin calcium: Design, formulation, bioavailability and pharmacokinetic evaluation. Colloids Surf. B: Biointerfaces 112:337-343

27. Andreas Hafner, Fritz Blatter, Martin Szelagiewicz, Bernd Siebenhaar (2016) Multicomponent system of rosuvastatin calcumi salt and sorbitol. Unites States patent US9.249.108 B2

28. Andreas Hafner, Fritz Blatter, Martin Szelagiewicz, Bernd Siebenhaar (2014) Multicomponent crystalline (52) u.s. cl. system of rosuvastatin calcum salt and vanillin. Unites States patent US 8.841.316 B2.

29. Andreas Hafner, Fritz Blatter, Martin Szelagiewicz, Bernd Siebenhaar (2014) Multicomponent crystalline system of rosuvastatin calcum salt and vanillin Unites States patent US 8.716.305 b2.
30. Clark Ferrari, Andrea Castellin, Marco Galvagni, Nicolas Tesson, Jordi De Mier (2014) Lloreng Rafecas. co-crystal intermediates of rosuvastatin and methods of using same. Unites States patent US 8.815862 b2.

31. Song Y, Wang LY, Liu F, Li YT, Wu ZY, Yan CW (2019) Simultaneously enhancing the in vitro/in vivo performances of acetazolamide using proline as a zwitterionic coformer for cocrystallization. Cryst Eng Comm 21:30643073

32. Nugrahani I, Utami D, Ibrahim S, Nugraha YP, Uekusa H (2018) Zwitterionic cocrystal of diclofenac and L-proline: Structure determination, solubility, kinetics of cocrystallization, and stability study. Eur J Pharm Sci 117:168-176

33. Tilborg A, Norberg B, Wouters J (2014) Pharmaceutical salts and cocrystals involving amino acids: A brief structural overview of the state-of-art. Eur J Med Chem 74:411-426

34. Tumanova N, Tumanov N, Robeyns K, Filinchuk Y, Wouters J, Leyssens T (2014) Structural insight into cocrystallization with zwitterionic co-formers: cocrystals of S-naproxen. Cryst Eng Comm 16:8185-8196

35. Yadav A, Shete A, Dabke A, Kulkarni P, Sakhare S (2009) Co-crystals: a novel approach to modify physicochemical properties of active pharmaceutical ingredients. Indian JPharm Sci 71:359

36. Shan N, Zaworotko MJ (2008) The role of cocrystals in pharmaceutical science. Drug Discov Today 13:440-446

37. Zhou Z, Tong HHY, Li L, Shek FLY, Lv Y, Zheng Y (2014) Synthesis, crystal structures and phase transformation of the new solid-state forms of tetrandrine. RSC Adv 4:62586-62593

38. Owoyemi BCD, Da Silva CCP, Souza MS, Diniz LF, Ellena J, Carneiro RL (2019) Fluconazole: synthesis and structural characterization of four new pharmaceutical cocrystal forms. Cryst Grow \& Design 19:648-657

39. Kumar M, Hirpara R, Manikkath J, Sivakumar K, Managuli RS, Gourishetti K, Krishnadas N, Shenoy RR, Jayaprakash B, Rao CM, Mutalik S (2017) Long circulating PEGylated-chitosan nanoparticles of rosuvastatin calcium: development and in vitro and in vivo evaluations. Int J Biol Macromol 107: 2190-2200

40. Elanthiraiyan M, Kandasamy M, Kanagan G, Govindarajan G, Pari S, Sambasivam R (2019) Growth and some characterization of L-asparagine monohydrate potassium iodide (LAMPI) crystals. Compliance Eng J 10:286-297

41. Pawlukojć A, Hołderna-Natkaniec K, Bator G, Natkaniec I (2014) L-glutamine: dynamical properties investigation by means of INS, IR, RAMAN, 'H NMR and DFT techniques. Chem Phys 443:17-25

42. Kapure VJ, Pande W, Deshmukh PK (2013) Dissolution enhancement of rosuvastatin calcium by liquisolid compact technique. J Pharm 2013:1-9, Article ID 315902. https://doi.org/10.1155/2013/315902

43. Mäder K, Mehnert W (2001) Solid lipid nanoparticles, production, characterization and applications. Adv Drug Deliv Rev 47:165-196

44. Beg S, Raza K, Kumar R, Chadha R, Katare OP, Singh B (2016) Improved intestinal lymphatic drug targeting via phospholipid complex-loaded nanolipospheres of rosuvastatin calcium. RSC Adv 6:8173-8187

45. Shete G, Puri V, Kumar L, Bansal AK (2010) Solid state characterization of commercial crystalline and amorphous atorvastatin calcium samples. AAPS PharmSciTech 11:598-609

46. Li HB, Niu R, Yang JL, Nie J, Yang DZ (2011) Photocrosslinkable tissue adhesive based on dextran. Carbohydr Polym 86:1578-1585

47. Desiraju GR (2013) Crystal engineering: from molecule to crystal. J Am Chem Soc 135:9952-9967

48. Sarfraz RM, Ahmad M, Mahmood A, Akram MR, Abrar A (2017) Development of $\beta$-cyclodextrin-based hydrogel microparticles for solubility enhancement of rosuvastatin: an in vitro and in vivo evaluation. Drug Des Devel Ther 11: 3083-3096

49. Duggirala NK, Perry ML, Almarsson Ö, Zaworotko MJ (2016) Pharmaceutical cocrystals: along the path to improved medicines. Chem Commun 52:640-655

50. Sun CC, Hou H (2008) Improving mechanical properties of caffeine and methyl gallate crystals by cocrystallization. Cryst Growth Des 8:1575-1579

51. Tanabe Y, Maeno Y, Ohashi K, Hisada H, Roy A, Carriere J, Heyler R, Fukami T (2019) Screening a trace amount of pharmaceutical cocrystals by using an enhanced nano-spot method. Eur J Pharm Biopharm 136:131-137

52. Shete A, Murthy S, Korpale S, Yadav A, Sajane S, Sakhare S, Doijad R (2015) Cocrystals of itraconazole with amino acids: screening, synthesis, solid state characterization, in vitro drug release and antifungal activity. J Drug Deliv Sci Tec 28:46-55

53. Luo Y, Chen S, Zhou J, Chen J, Tian L, Gao LX, Zhang Y, Ma A, Li L, Zhou Z (2019) Luteolin cocrystals: characterization, evaluation of solubility, oral bioavailability and theoretical calculation. J Drug Deliv Sci Tec 50:248-254 
54. Inouye S, litaka Y (1964) Crystallographic data on the molecular complexes of tetracycline salts. Acta Crystallogr 17:207e208

55. Rajagopal K, Krishnakumar RV, Nandhini MS, Natarajan S (2003) L-Histidinium hemihydrochloride tartrate tartaric acid dehydrate. Acta Crystallogr Sect E Struct Rep Online 59:0955eo958

56. Perlovich GL (2015) Thermodynamic characteristic of cocrystal formation and melting points for rational design of pharmaceutical two-component systems. Cryst Eng Comm 17:7019-7028

57. Rustichelli C, Gamberini G, Ferioli V, Gamberini MC, Ficarra R, Tommasini S (2000) Solid-state study of polymorphic drugs: carbamazepine. J Pharm Sci 23:41-45

58. Unsalan O, Erdogdu Y, Gulluoglu MT (2009) FT-Raman and FT-IR spectral and quantum chemical studies on some flavonoid derivatives: baicalein and naringenin. J Raman Spectrosc 40:562-570

59. Chadha R, Bhalla Y, Nandan A, Chadha K, Karan M (2017) Chrysincocrystals: characterization and evaluation. J Pharm Biomed Anal 134:361-371

60. Alatas F, Ratih H, Soewandhi SN (2015) Enhancement of solubility and dissolution rate of telmisartan by telmisartan-oxalic acid cocrystal formation. Int J Pharma Sci 7:5-8

61. Cheney ML, Weyna DR, Shan N, Hanna M, Wojtas L, Zaworotko MJ (2011) Coformer selection in pharmaceutical cocrystal development: a case study of a meloxicam aspirin cocrystal that exhibits enhanced solubility and pharmacokinetics. J Pharmaceut Sci 100:2172-2181

62. Zhang GGZ, Law D, Schmitt EA, Qiu Y (2004) Phase transformation considerations during process development and manufacture of solid oral dosage forms. Adv Drug Deliv Rev 56(3):371-390

63. Vemuri VD, Lankalapalli S (2019) Insight into concept and progress on pharmaceutical co-crystals: an overview. Indian J Pharmaceut Educ Res 53(4s)::5522-s538

64. Dnyaneshwar P, KaleSandeep S, ZodeArvind K, Bansal (2017) Challenges in Translational Development of Pharmaceutical Cocrystals. J Pharm Sci 106: $457-470$

\section{Publisher's Note}

Springer Nature remains neutral with regard to jurisdictional claims in published maps and institutional affiliations.

\section{Submit your manuscript to a SpringerOpen ${ }^{\circ}$ journal and benefit from:}

- Convenient online submission

- Rigorous peer review

- Open access: articles freely available online

- High visibility within the field

- Retaining the copyright to your article

Submit your next manuscript at $\boldsymbol{\nabla}$ springeropen.com 